\title{
Investigation of heterogeneous solid acid catalyst performance on low grade feedstocks for biodiesel production: a review
}

\begin{abstract}
The conventional fossil fuel reserves are continually declining worldwide and therefore posing greater challenges to the future of the energy sources. Biofuel alternatives were found promising to replace the diminishing fossil fuels. However, conversion of edible vegetable oils to biodiesel using homogeneous acids and base catalysts is now considered as indefensible for the future particularly due to food versus fuel competition and other environmental problems related to catalyst system and feedstock. This review has discussed the progression in research and growth related to heterogeneous catalysts used for biodiesel production for low grade feedstocks. The heterogeneous base catalysts have revealed effective way to produce biodiesel, but it has the limitation of being sensitive to high free fatty acid (FFA) or low grade feedstocks. Alternatively, solid acid catalysts are capable of converting the low grade feedstocks to biodiesel in the presence of active acid sites. The paper presents a comprehensive review towards the investigation of solid acid catalyst performance on low grade feedstock, their category, properties, advantages, limitations and possible remedy to their drawbacks for biodiesel production.
\end{abstract}

Keyword: Fatty acid methyl ester; Heterogeneous catalyst; Low grade feedstock; Esterification; Transesterification 